Please do not remove this page

RMIT

UNIVERSITY

\title{
Observation of surface gap solitons
}

Rosberg, Christian; Neshev, Dragomir; Krolikowski, Wieslaw; Kivshar, Yuri; Mitchell, Arnan; Vincencio, Rodrigo; Molina, Mario

https://researchrepository.rmit.edu.au/esploro/outputs/9921860176901341/filesAndLinks?institution=61RMIT_INST\&index=null

Rosberg, C., Neshev, D., Krolikowski, W., Kivshar, Y., Mitchell, A., Vincencio, R., \& Molina, M. (2006).

Observation of surface gap solitons. Proceedings of the Conference on Lasers and

Electro-Optics/Quantum Electronics and Laser Science Conference, 1-2.

https://doi.org/10.1109/CLEO.2006.4627794

Published Version: https://doi.org/10.1109/CLEO.2006.4627794

Repository homepage: https://researchrepository.rmit.edu.au

(C) 2006 IEEE

Downloaded On 2023/04/26 23:55:18 +1000

Please do not remove this page 


\title{
CMK7.pdf
}

\section{Observation of surface gap solitons}

\author{
Christian R. Rosberg, Dragomir N. Neshev, Wieslaw Krolikowski, and Yuri S. Kivshar \\ Nonlinear Physics Centre and Laser Physics Centre, \\ Centre for Ultrahigh-bandwidth Devices for Optical Systems (CUDOS), \\ Research School of Physical Sciences and Engineering, \\ Australian National University, ACT 0200 Canberra, Australia \\ Home page: http://www.rsphysse.anu.edu.au/nonlinear, E-mail: crr124@rsphysse.anu.edu.au
}

\author{
Arnan Mitchell \\ School of Electrical and Computer Systems Engineering, RMIT University, Melbourne, Australia \\ Rodrigo A. Vicencio \\ Max-Planck-Insitut für Physik komplexer Systeme, Dresden, Germany \\ Mario I. Molina \\ Departamento de Física, Facultad de Ciencias, Universidad de Chile, Santiago, Chile
}

\begin{abstract}
We predict, in the framework of a nonlinear discrete model, and demonstrate experimentally in defocusing waveguide arrays, that self-localization near the edge of a photonic lattice can result in the formation of surface gap solitons.
\end{abstract}

(C) 2005 Optical Society of America

OCIS codes: 190.4420; 190.5940;

Surface modes are a special type of waves localized at an interface between two different media. In periodic systems, staggered modes localized at the surface are known as Tamm states ${ }^{1}$, first found as localized electronic states at the edge of a truncated periodic potential. Surface states have been studied in different fields of physics, including optics ${ }^{2}$ where such waves are confined to the interface between periodic and homogeneous dielectric media. Recently it was predicted theoretically and demonstrated experimentally that nonlinear self-trapping of light near the edge of a waveguide array with self-focusing nonlinearity can lead to the formation of discrete surface solitons ${ }^{3,4}$. However, when light propagates in a defocusing nonlinear medium with periodically modulated refractive index, self-localization can occur in the form of surface modes having a staggered phase structure, thus enabling a full analogy with the localized electronic surface Tamm states.

In this work, we study the formation of solitons near the edge of a defocusing nonlinear medium with a semi-infinite periodic refractive index modulation in the transverse direction. We demonstrate that a simple nonlinear discrete model can be used to predict a set of self-localized surface modes resulting from the interaction of a gap soliton ${ }^{5}$ with the interface, and to explain the existence and stability of multiple stationary states formed near the surface. In experiment, we observe for the first time the formation of staggered surface gap solitons in a $\mathrm{LiNbO}_{3}$ waveguide array with defocusing nonlinearity.
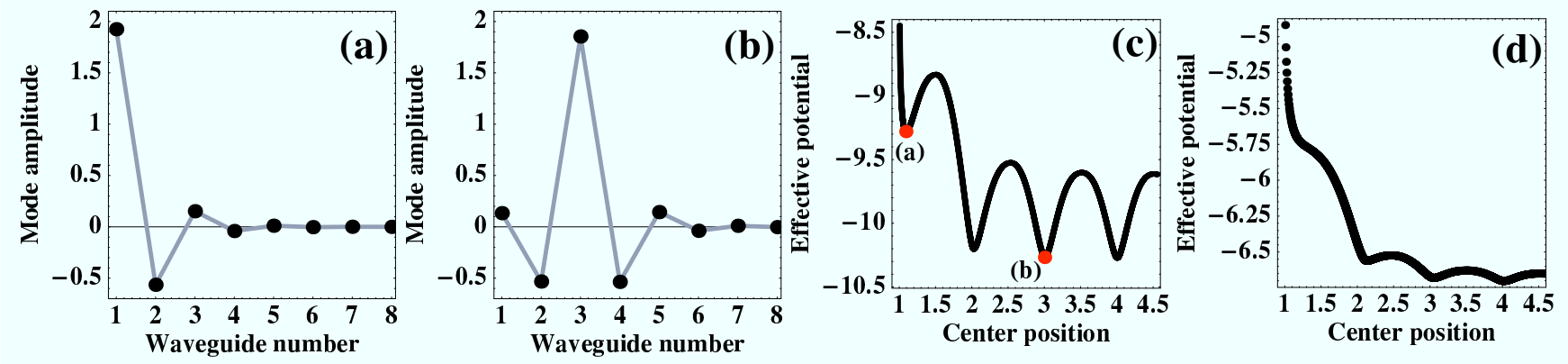

FIG. 1: (a,b) Examples of two near-surface gap states found as the localized solutions of a discrete model with defocusing nonlinearity. The surface mode (a) becomes stable only above a certain power threshold. (c,d) Effective interaction energy of the gap soliton with the surface and the lattice above and below the threshold power, respectively. Marked minima in (c) correspond to the stationary states in $(\mathrm{a}, \mathrm{b})$.

First we study a nonlinear model with defocusing nonlinearity which, in the case of weak coupling between the neighboring waveguides, can be reduced to a discrete model. Our analysis reveals that the combined effect of the

Citation: Rosberg, C, Neshev, D, Krolikowski, W, Kivshar, Y, Mitchell, A, Vincencio, R and Molina, M 2006, 'Observation of surface gap solitons', in Proceedings of the Conference on Lasers and ElectroOptics/Quantum Electronics and Laser Science Conference, Long Beach, California, USA, $21-26$ May 2006. 


\section{CMK7.pdf}

repulsive surface potential and the lattice discreteness results in the existence of families of self-trapped gap states localized near the surface. Figures $1(a, b)$ show the discretized profiles of two staggered surface states. A detailed analysis of the stationary states and their transformations by a numerical constraint method allows to obtain the effective energy that describes both the mode interaction with the surface and the trapping by the discrete lattice. Stable stationary states, corresponding to the minima of this function, are found to appear only above a certain power threshold. Figures 1(c,d) show the effective potential for the two cases, above and below the threshold, respectively. The self-localized staggered surface modes described here can be classified as nonlinear optical Tamm states.
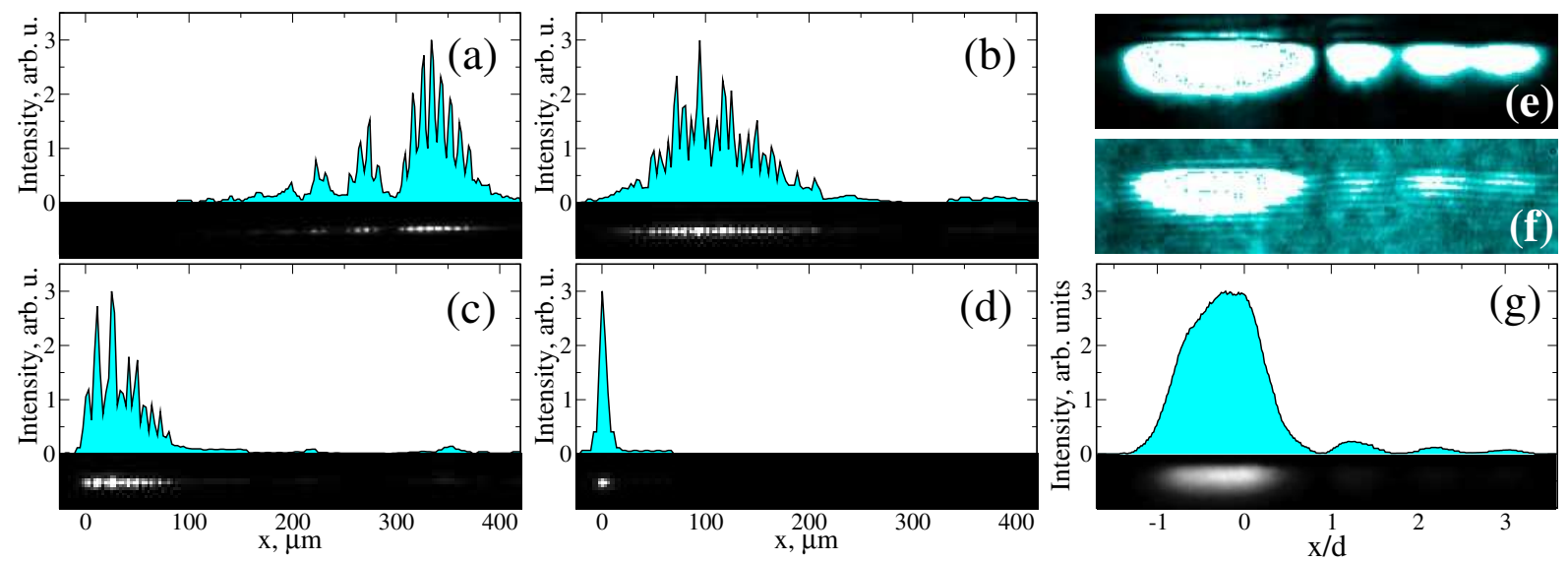

FIG. 2: Experimental formation of surface gap solitons in a defocusing waveguide array. (a) Intensity distribution and corresponding intensity profile at the output of the array at low power $(\sim 0.8 \mu \mathrm{W})$ beam experiencing linear diffraction. Array's edge and input position is located at $x=0$. (b-d) Output in the nonlinear regime 30, 90 and 240 seconds, respectively, after the input power is increased to $100 \mu \mathrm{W}$. (e-g) Zoom on localized surface state in (d). Saturated image (e) and interferogram (f) confirming its staggered phase structure.

In experiment, we study the formation of surface gap solitons in an array of closely spaced single mode waveguides, fabricated by Titanium indiffusion in a mono-crystal lithium niobate wafer. The refractive index contrast is $\Delta n=10^{-4}$, the waveguide spacing $d=9 \mu \mathrm{m}$, and the sample length $50 \mathrm{~mm}$. The LiNbO 3 sample exhibits a strong photovoltaic effect which leads to a self-defocusing nonlinearity. The waveguide array is externally illuminated with white light during the experiment in order to reduce the nonlinear response time. At the input face of the sample, an extraordinarily polarized probe beam from a cw Nd:YVO laser at wavelength $\lambda=532 \mathrm{~nm}$ is tightly focused onto the first waveguide at the edge of the array, and imaged onto a CCD camera at the output. At low laser power $(\sim 0.8 \mu \mathrm{W})$ the probe beam experiences discrete diffraction as shown in Fig. 2(a). At the output the beam profile spans about ten waveguides, and it is centered approximately 37 lattice sites from the input excitation point $(x=0$ at the edge of the array). Increasing the laser power to $100 \mu \mathrm{W}$ induces a strong nonlinear response which results in single-site localization at the surface waveguide [Fig. 2(d)], in a similar way as in self-focusing waveguide arrays. The effect of the photovoltaic nonlinearity is of rather slow nature and the total time for localization onto the surface takes approximately 10min. Monitoring the temporal dynamics of the soliton formation provides additional information about the process of localization. This dynamics is depicted in Figs. 2(b-d) which show snapshots of the output intensity distribution at times 30, 90, and 240 seconds after the power has been increased. Figures 2(e-g) show a zoom on the localized surface state in (d). We verify its staggered phase structure by saturated image detection of zero intensity lines [Fig. 2(e)] and by the interferometric measurement with a plane reference wave [Fig. 2(f)].

In conclusion, we have predicted theoretically and demonstrated in experiment that gap solitons can be stabilized near the surface of a periodic medium with self-defocusing nonlinearity in the form of staggered surface modes, providing the first evidence of an optical analog of nonlinear surface Tamm states.

\section{References}

[1] I. E. Tamm, Z. Phys. 76, 849 (1932).

[2] P. Yeh, A. Yariv, and A. Y. Cho, Appl. Phys. Lett. 32, 102 (1978).

[3] K. G. Markis, S. Suntsov, D. N. Christodoulides, G. I. Stegeman, and A. Hache, Opt. Lett. 30, 2466 (2005).

[4] S. Suntsov, K. G. Makris, D. N. Christodoulides, G. I. Stegeman, in Nonlinear Guided Waves and Their Applications Topical Meeting (The Optical Society of America, Washington, DC, 2005), ThC4.

[5] Yu. S. Kivshar and G. P. Agrawal, Optical Solitons: From Fibers to Photonic Crystals (Academic Press, San Diego, 2003). 